\title{
Are pedigree-based mutation rates the Rosetta Stone of molecular ecology? Promises and pitfalls of whole genome comparisons among closely related individuals.
}

\author{
Anne Yoder $^{1}$ and George Tiley ${ }^{1}$ \\ ${ }^{1}$ Duke University
}

January 22, 2021

\begin{abstract}
Germline mutations are the raw material for natural selection, driving species evolution and the creation of earth's biodiversity. Life on earth would stagnate without this driver of genetic diversity. Yet, it is a double-edged sword. An excess of mutations can have devastating effects on fitness and population viability. It is therefore one of the great challenges of molecular ecology to determine the rate and spectrum by which these mutations accrue across the tree of life. Advances in high-throughput sequencing are providing new opportunities for characterizing these rates and patterns within species and populations, thus informing essential evolutionary parameters such as the timing of speciation events, the intricacies of historical demography, and the degree to which lineages are subject to the burdens of mutational load. Here, we will focus on the applications and limitations of whole-genome comparisons among closely related individuals in what are typically described as "trio" analyses for the detection of germline mutations as they arise in real time. By sequencing and comparing whole-genomes generated for individuals of known relatedness - typically, parent to offspring - investigators can ideally count and characterize mutations as they appear per generation. The promise for gaining insight into classic hypotheses of molecular evolution is high, though so too is the cost. Namely, the technical challenges are daunting given that pedigree-based studies are essentially searching for needles in a haystack. Even so, the opportunities are so enticing, and the field so young, we can say with confidence that fundamental insights have only just begun to emerge.
\end{abstract}

\section{Hosted file}

Yoder\&Tiley_MolEcol.2021.pdf available at https://authorea.com/users/391165/articles/505341are-pedigree-based-mutation-rates-the-rosetta-stone-of-molecular-ecology-promises-andpitfalls-of-whole-genome-comparisons-among-closely-related-individuals

\section{Hosted file}

Yoder\&Tiley.Table_1.pdf available at https://authorea.com/users/391165/articles/505341are-pedigree-based-mutation-rates-the-rosetta-stone-of-molecular-ecology-promises-andpitfalls-of-whole-genome-comparisons-among-closely-related-individuals 


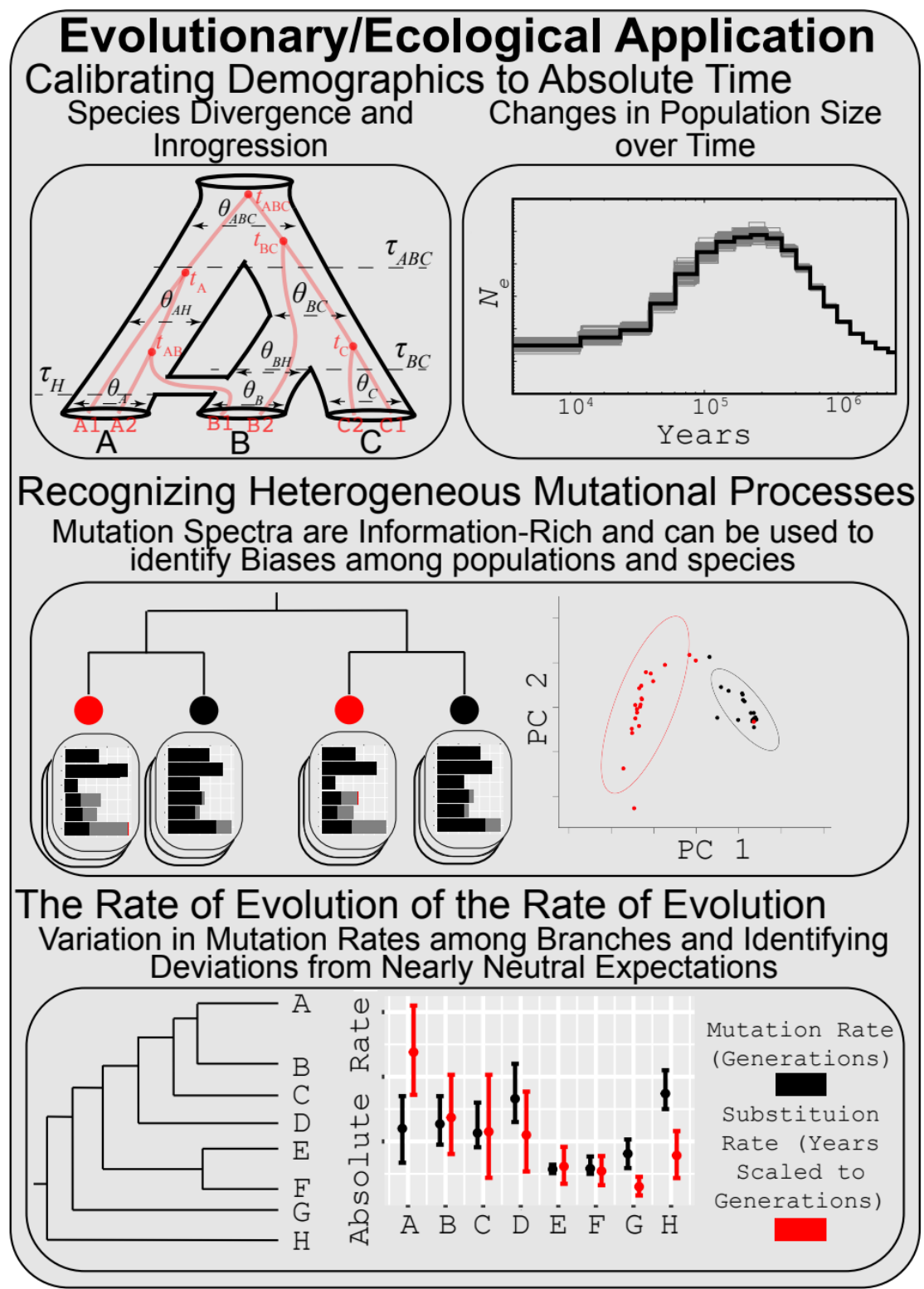




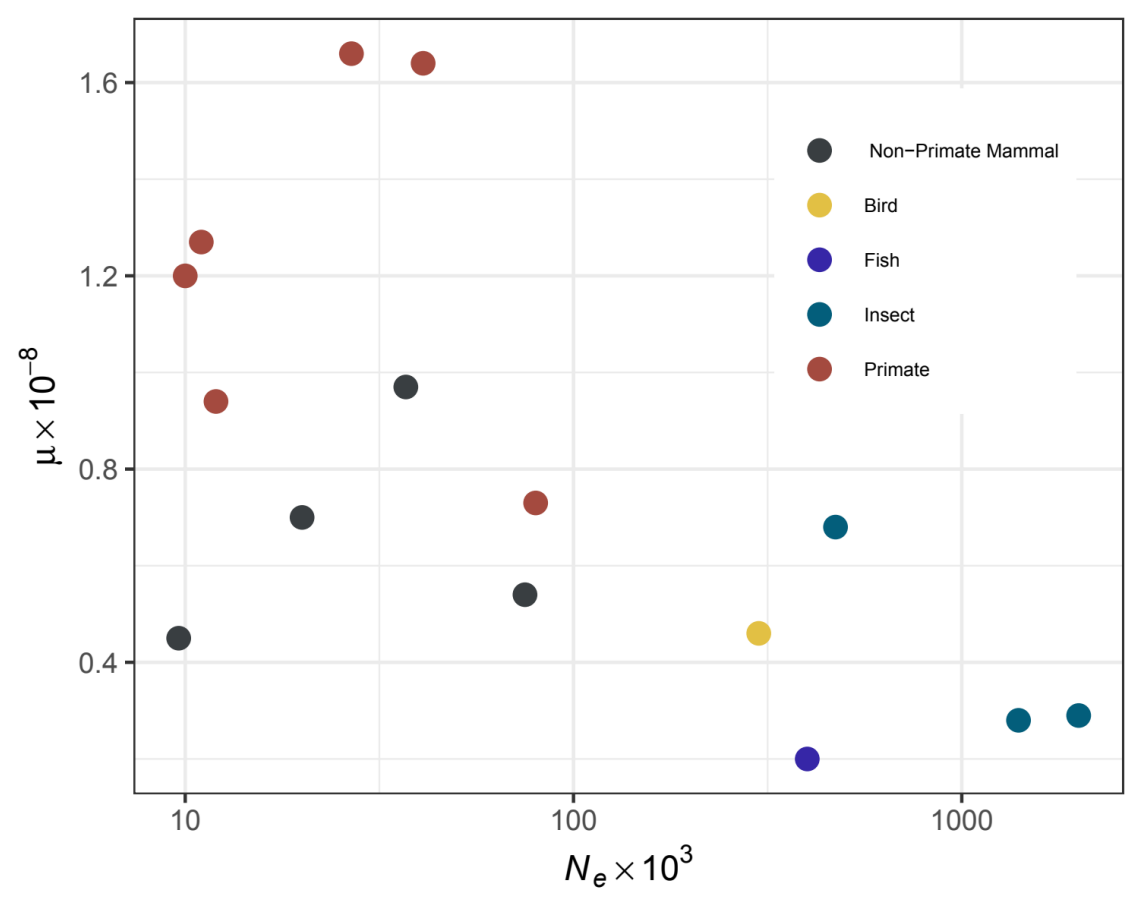




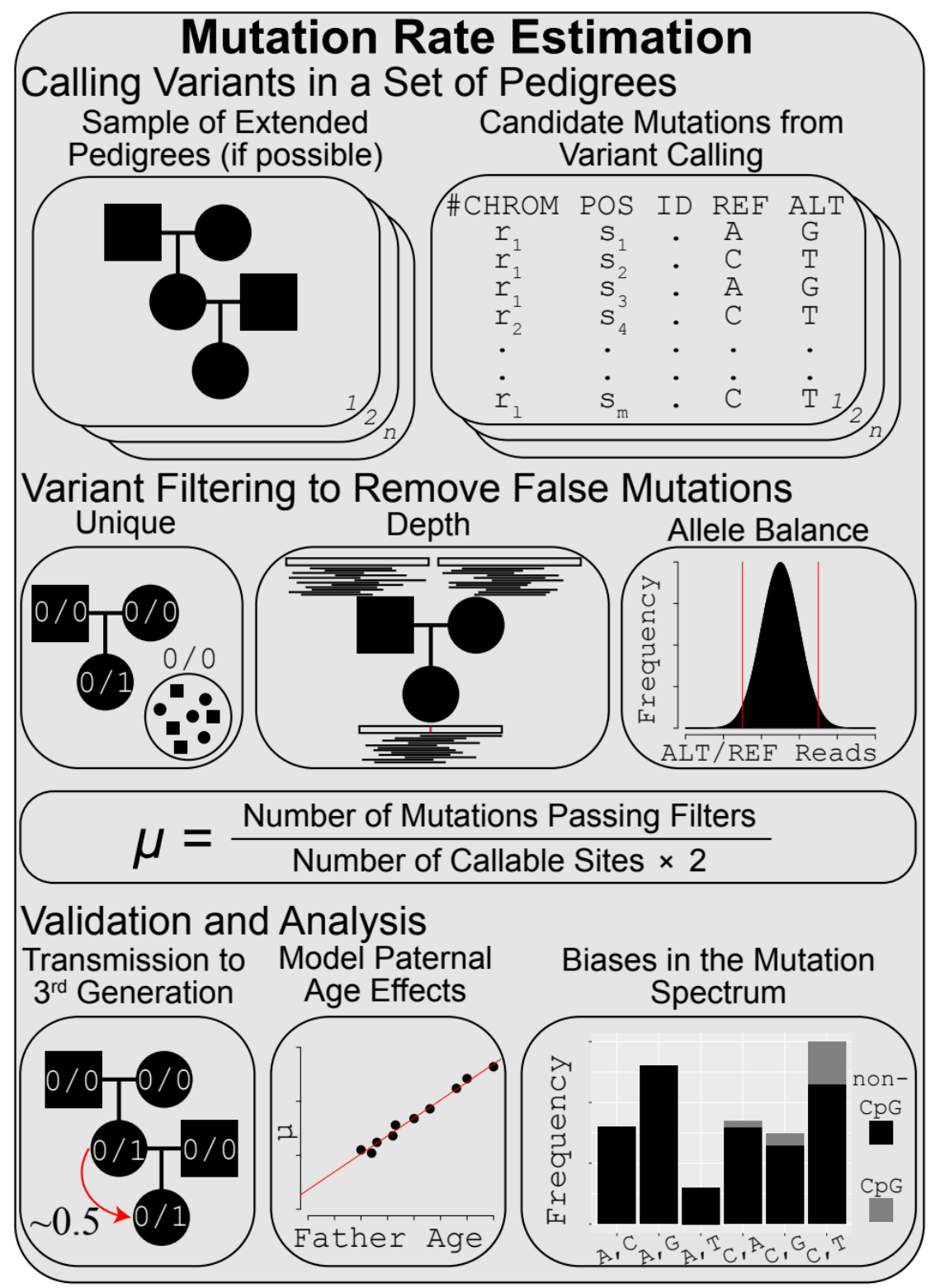

